\title{
Higher Order Weight Functions in Fracture Mechanics of Multimaterials
}

\author{
A. Yu. Belov \\ Additional information is available at the end of the chapter
}

http://dx.doi.org/10.5772/55360

\section{Introduction}

The quantities characterizing near-tip fields of cracks are generally recognized to play a crucial role in both linear and nonlinear fracture mechanics. Among various methods developed to analyze the structure of the near-tip fields, the weight function technique of Bueckner $[4,6]$ based on Betti's reciprocity theorem turned out to be especially promising. The concept of higher-order weight functions in mechanics of elastic cracks was introduced by Sham [20,21] as an extension of the weight function approach. A historical introduction into the existing alternative formulations of the weight function theory and a review of its earlier development can be found in the papers by Belov and Kirchner [28, 31]. The theory of weight functions treats the stress intensity factor $K$, which is a coefficient normalizing the stress singularity $\sigma=K /(2 \pi r)^{1 / 2}$ at the crack tip, as a linear functional of loadings applied to an elastic body. The kernel of the functional is however independent of loadings and, in this sense, universal for the given body geometry and crack configuration. To emphasize this fact, Bueckner [4] suggested that the kernel to be called 'universal weight function'. The weight functions play the role of influence functions for stress intensity factors, since the weight function value at a point situated inside the body or at its surface (including crack faces) is equal to the stress intensity factor, which is due to the unit concentrated force applied at this point. The weight function based functionals can be constructed not only for external forces but also for the dislocation distributions described by the dislocation density tensor, as it was shown by Kirchner [14]. The objective of the weight function theory is not to compute complete stress distributions in cracked bodies for an arbitrary loading, but to express only one parameter $K$ characterizing the strength of the near-tip stress field as a functional (weighted average) of the loading. In particular, in the simplest case of a cracked body subjected to only surface loadings the functional has the form of a contour integral. However, in order to apply the weight function theory to practical situations, the kernel of the functional has to be evaluated and this can be done by solving a special elasticity problem, for instance, numerically by a finite element method. The stress singularities are inherent not only to cracks. Sharp 
re-entrant corners or notches that are encountered in a number of engineering structures can become likely sites of stress concentrations and therefore the potential sources of the crack initiation. At the tips (or vertices) of these notches the stress can be also singular $\sigma=K / r^{1-s}$, where $s>0$ and $K$ is a generalized stress intensity factor normalizing the stress singularity. An attractive feature of the approach based on Betti's reciprocity theorem is that it enables for the weight functions to be constructed not only for sharp cracks but also for notches of finite opening angle [19]. It is the purpose of this paper to review the main ideas underlying the higher-order weight function methodology and to consider its applications to elastically anisotropic multimaterials with notches or cracks. The present analysis is confined to the two-dimensional structures in the state of the generalized plane deformation, where considerable analytical advancement was demonstrated in the last two decades.

As is known, the stress field in a finite two-dimensional elastic body containing an edge crack can be represented in series form over homogeneous eigenfunctions of an infinite plane with a semi-infinite crack. Such a series representation was first utilized by Williams $[1,2]$ to describe stress distributions around the crack tip, and is commonly referred to as Williams' eigenfunction expansion, although Williams confined himself only to the case of isotropy and spacial homogeneity of the elastic constants tensor. The eigenfunction expansion of this type however exists whatever the body is elastically isotropic or anisotropic, homogeneous or angularly inhomogeneous (with elasticity constants dependent on the azimuth around the crack tip). The weight functions introduced by Bueckner enable to evaluate only the stress intensity $K$, that is the magnitude of the singular term, which close to the crack tip dominates other terms in the Williams' expansion. It is the purpose of higher order weight function theory to evaluate coefficients of non-singular terms in this expansion.

\section{Symmetry in anisotropic theory of elasticity}

If one exploits the linear elasticity theory, the tensor of the second order elastic constants $C_{i j k l}(\mathbf{r})$ of an anisotropic medium (both homogeneous and inhomogeneous) possesses the following types of symmetry:

a) due to the symmetry of stresses and strains

$$
C_{i j k l}(\mathbf{r})=C_{j i k l}(\mathbf{r})=C_{i j l k}(\mathbf{r})
$$

b) due to the existence of the elastic potential $W\left(\varepsilon_{k l}\right)$

$$
C_{i j k l}(\mathbf{r})=C_{k l i j}(\mathbf{r})
$$

Owing to both properties given in Eqs. (1)-(2), one has

$$
(a b)=(b a)^{\mathrm{t}},
$$

where the real $3 \times 3$ matrices are constructed according to the rule

$$
(a b)_{j k}=a_{i} C_{i j k l}(\mathbf{r}) b_{l}
$$


for two arbitrary vectors $a_{i}$ and $b_{l}$. Although Eq. (3) looks rather simple, it underlies many fundamental results of the anisotropic elasticity theory. In particular, the proof of the orthogonality relation for the six-dimensional Stroh eigenvectors [3] is based only on Eq. (3), see [22] for further details. Here it is worth to mention that Betti's reciprocity theorem is based on the symmetry properties (1)-(2) as well. This fact was utilized in [5] to derive the aforementioned orthogonality relation for the Stroh eigenvectors from Betti's theorem. In fact, practically all significant analytical achievements in the anisotropic theory of elasticity employ the directly following from Eq. (3) symmetry relation

$$
(\hat{\mathbf{T}} \hat{\mathbf{N}})^{\mathrm{t}}=\hat{\mathbf{T}} \hat{\mathbf{N}},
$$

where the $6 \times 6$ matrix $\hat{\mathrm{T}}$ is defined as

$$
\hat{\mathbf{T}}=\left(\begin{array}{ll}
0 & \mathbf{I} \\
\mathbf{I} & 0
\end{array}\right), \quad \hat{\mathbf{T}}^{2}=\left(\begin{array}{ll}
\mathbf{I} & 0 \\
0 & \mathbf{I}
\end{array}\right)=\hat{\mathbf{I}},
$$

$\hat{\text { I }}$ is the $6 \times 6$ unit matrix, and the $6 \times 6$ matrix $\hat{\mathbf{N}}=\hat{\mathbf{N}}(\mathbf{r})$ is the well-known matrix of Stroh

$$
\hat{\mathbf{N}}(\mathbf{r})=\left(\begin{array}{cc}
\hat{\mathbf{N}}_{1} & \hat{\mathbf{N}}_{2} \\
\hat{\mathbf{N}}_{3} & \hat{\mathbf{N}}_{4}
\end{array}\right)
$$

consisting of the $3 \times 3$ blocks

$$
\begin{array}{ll}
\hat{\mathbf{N}}_{1}=-(n n)^{-1}(n m), & \hat{\mathbf{N}}_{2}=-(n n)^{-1}, \\
\hat{\mathbf{N}}_{3}=(m m)-(m n)(n n)^{-1}(n m), \hat{\mathbf{N}}_{4}=-(m n)(n n)^{-1} .
\end{array}
$$

The blocks of the Stroh matrix are formed by a convolution of the elastic constants tensor $C_{i j k l}(\mathbf{r})$ with two unit vectors $\mathbf{m}$ and $\mathbf{n}$ forming together with the unit vector $\mathbf{t}$ the right-handed basis $(\mathbf{m}, \mathbf{n}, \mathbf{t})$. Eq. (5) is easily proved by direct inspection.

\section{The consistency equation}

Here, we review the fundamentals of the weight function theory in inhomogeneous elastic media, following the method of Belov and Kirchner [28]. Let us consider a two-dimensional (that is infinite along the axis $x_{3}$ ) notched body $A$ of finite size in the $x_{1} x_{2}$-plane, as shown in Fig. 1. The body is supposed to be loaded such that a state of generalized plane strains occurs, that is the displacement vector $\mathbf{u}$ remains invariant along $x_{3}$ and has both plane $\left(u_{1}\right.$ and $\left.u_{2}\right)$ and anti-plane $\left(u_{3}\right)$ components. We deal with a special class of multimaterials, which are composed from the elastically anisotropic homogeneous wedge-like regions with a common apex, as shown Fig. 2. The wedges differ in their elastic constants. In fact, the multimaterial structures discussed in this chapter are a particular case of the elastic media with angular inhomogeneity of the elastic properties. Therefore they can be treated within the framework of the general formalism developed by Kirchner [17] for elastically anisotropic angularly inhomogeneous media, where the elasticity constants $C_{j i k l}(\omega)$ depend on the azimuth $\omega$ counted around the axis $x_{3}$ from which the radius $r$ is counted, as illustrated in Fig. 3. The essence of this approach is to employ a six-dimensional consistency equation for the field variable $(\mathbf{u}, \phi)$ formed by the displacement vector $\mathbf{u}$ and the Airy stress function vector $\boldsymbol{\phi}$. The 


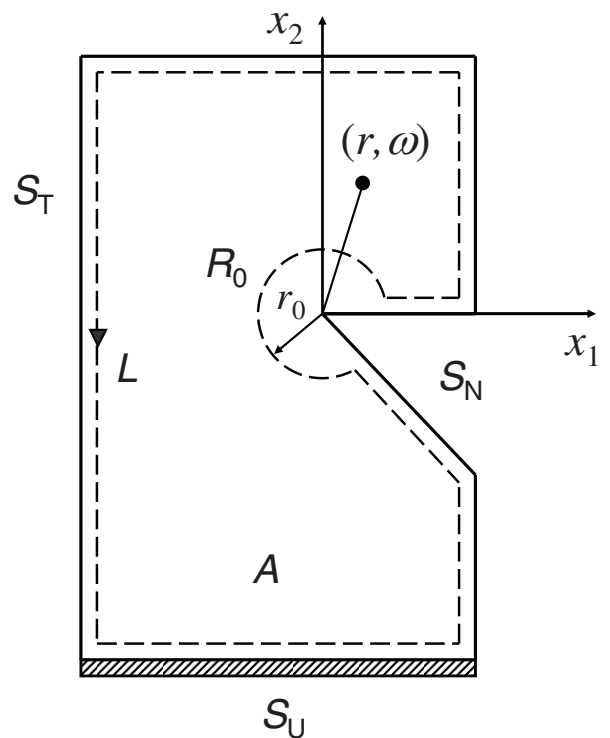

Figure 1. Finite specimen $A$ with a notch. The tractions are prescribed on $S_{\mathrm{T}}$ and the displacements on $S_{\mathrm{U}}$; the notch faces $S_{\mathrm{N}}$ are traction free. The reciprocity theorem is applied to the dashed contour $L$.

consistency equation results from the fact that some linear forms consisting of the first-order spacial derivatives of the displacements and stress functions must represent components of the same stress tensor. Consequently the stresses $\sigma_{i j}$ can be equally derived from $\mathbf{u}$ via Hook's law as

$$
\sigma_{i j}=C_{i j k l}(\omega) \partial_{k} u_{l}
$$

or from $\phi$ according to

$$
\sigma_{i 1}=\partial_{2} \phi_{i}, \quad \sigma_{i 2}=-\partial_{1} \phi_{i} .
$$

Direct comparison of Eq. (9) and Eq. (10) yields a first-order differential equation

$$
\left\{\hat{\mathbf{N}}(\omega) \frac{\partial}{\partial r}-\hat{\mathbf{I}} \frac{1}{r} \frac{\partial}{\partial \omega}\right\}\left(\begin{array}{l}
\mathbf{u}(r, \omega) \\
\boldsymbol{\phi}(r, \omega)
\end{array}\right)=\mathbf{0}
$$

where the matrix $\hat{\mathbf{N}}(\omega)$ is defined by Eq. (7) and Eq. (8) and the unit vectors $\mathbf{m}$ and $\mathbf{n}$ are rotated counterclockwise by an angle $\omega$ against a fixed basis $\left\{\mathbf{m}_{0}, \mathbf{n}_{0}\right\}$, as shown in Fig. 3. The consistency condition given in Eq. (11) ensures that any its solution corresponds to equilibrated stresses (because they are derived from the stress functions) and compatible strains (because they are derived from the displacements). Therefore, the solutions of Eq. (11) describe states free of body forces and dislocation distributions. As it was emphasized in [28], the consistency equation (11) remains valid for arbitrary inhomogeneity, where the matrix $\hat{\mathbf{N}}(r, \omega)$ depends also on the radius $r$ via $C_{i j k l}(r, \omega)$, and provides an extension of the well-known result [9] obtained under assumption of elastic homogeneity. The examples of successful application of the consistency equation to the analysis of the stress state due to linear defects such as dislocations, line forces, and disclinations in angularly inhomogeneous 


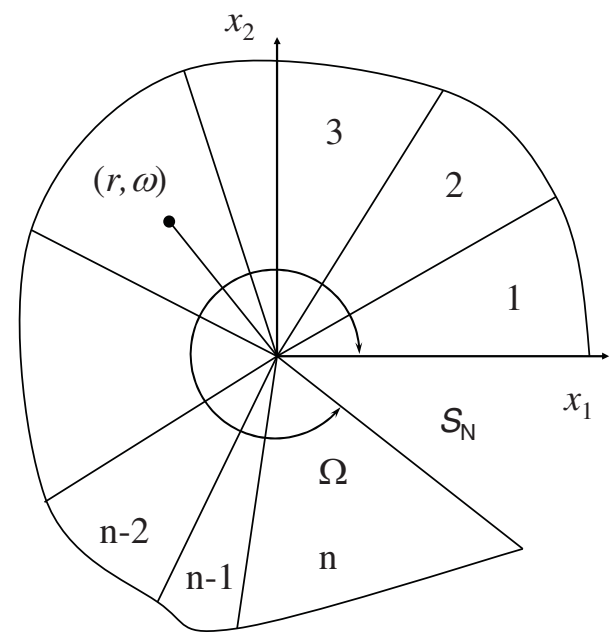

Figure 2. Multimaterial consisting of $n$ bonded together elastic wedges with different elastic constants $C_{i j k l}^{(m)},(m=1, \ldots, n)$.

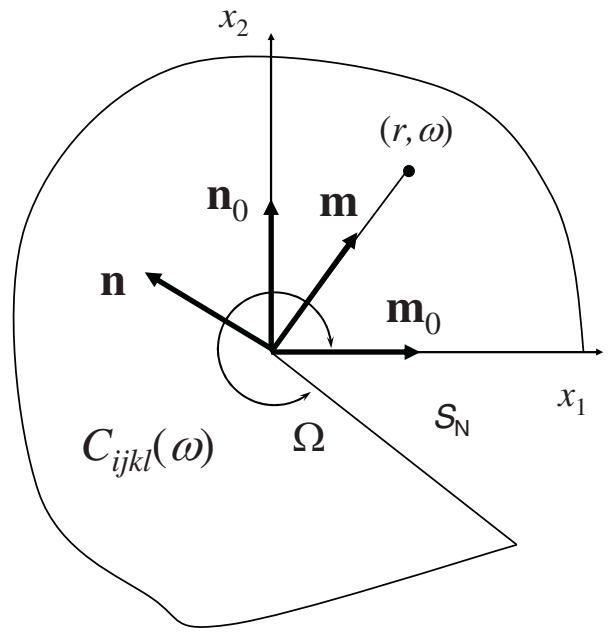

Figure 3. Elastic plane with a notch and the elastic constants $C_{i j k l}(\omega)$ continuously dependent on the azimuth $\omega$. Basis $(\mathbf{m}, \mathbf{n}, \mathbf{t})$ is rotated counterclockwise by an angle $\omega$ against a fixed basis $\left(\mathbf{m}_{0}, \mathbf{n}_{0}, \mathbf{t}\right)$.

anisotropic media can be found in [16] and [23, 24], respectively. The consistency equation was further applied in [29] to study the stress behavior in the angularly inhomogeneous elastic wedges near and at the critical wedge angle.

\section{Eigenfunction expansions}

According to [28], an extension of the Williams' eigenfunction expansion [1] to the notched body shown in Fig. 3 can be constructed from homogeneous solutions of Eq. (11). A suitable 
separable solution varying as a power of distance $r$ has the form

$$
\left(\begin{array}{l}
\mathbf{u}(r, \omega) \\
\boldsymbol{\phi}(r, \omega)
\end{array}\right)=r^{s} \hat{\mathbf{V}}^{(s)}(\omega)\left(\begin{array}{l}
\mathbf{h} \\
\mathbf{g}
\end{array}\right)
$$

where $(\mathbf{h}, \mathbf{g})$ is a constant six-dimensional vector and

$$
\hat{\mathbf{V}}^{(s)}(\omega)=\left(\begin{array}{cc}
\hat{\mathbf{V}}_{1}^{(s)} & \hat{\mathbf{V}}_{2}^{(s)} \\
\hat{\mathbf{V}}_{3}^{(s)} & \hat{\mathbf{V}}_{4}^{(s)}
\end{array}\right),
$$

is a $6 \times 6$ matrix function of the azimuth $\omega$, which is sometimes also referred to as transfer matrix. It is to be found by inserting the separable solution (12) into the consistency equation (11). As was shown in [17], this procedure results in the first-order ordinary differential equation

$$
\frac{d \hat{\mathbf{V}}^{(s)}(\omega)}{d \omega}=s \hat{\mathbf{N}}(\omega) \hat{\mathbf{V}}^{(s)}(\omega)
$$

with the initial condition

$$
\hat{\mathbf{V}}^{(s)}(0)=\hat{\mathbf{I}} \text {. }
$$

The solution of Eq. (14) is the ordered exponential defined as

$$
\begin{aligned}
\hat{\mathbf{V}}^{(s)}(\omega) & =\operatorname{Ordexp}\left(s \int_{0}^{\omega} \hat{\mathbf{N}}(\theta) \mathrm{d} \theta\right) \\
& =\prod_{i=1}^{k} \exp \left(s \hat{\mathbf{N}}\left(\theta_{i-1}\right) \delta \theta\right),
\end{aligned}
$$

where $\theta_{0}=0, \delta \theta=\omega / k$, and $k \rightarrow \infty$. A representation of the ordered exponential as a series expansion useful for approximate calculations within the framework of the perturbation theory can be found in [17]. The six-dimensional field (12) satisfies the consistency equation (11) and provides the solution, which is both compatible and equilibrated in the bulk for any value of the parameter s, which may be real or complex. Hence, the bulk operator $\hat{\mathbf{N}}(\omega)$ in Eq. (11) itself doesn't impose any restrictions on the admissible values of $s$. However, in order for (12) to become an eigenfunction of the angularly inhomogeneous notched plane (see Fig. 3), the appropriate boundary condition at the notch faces must be satisfied, and it is the boundary condition that results in the discrete spectrum of the eigenvalues $\left\{s_{n}\right\}$ and the corresponding eigenvectors $\left(\mathbf{h}_{n}, \mathbf{g}_{n}\right)$. If both notch faces, $\omega=0$ and $\omega=\Omega$, are traction free, the boundary condition reads as

$$
\boldsymbol{\phi}(r, 0)=\boldsymbol{\phi}(r, \Omega)=\mathbf{0} .
$$

In view of Eq. (15), the condition at the notch face $\omega=0$ implies that $\mathbf{g}=\mathbf{0}$. The condition at the other face, $\omega=\Omega$, gives a linear homogeneous algebraic system of equations for the three components of $\mathbf{h}$,

$$
\hat{\mathbf{V}}_{3}^{(s)}(\Omega) \cdot \mathbf{h}=\mathbf{0} .
$$

The system (18) has a non-trivial solution for $\mathbf{h}$ only provided that the parameter $s$ satisfies the eigenvalue equation

$$
\left\|\hat{\mathbf{V}}_{3}^{(s)}(\Omega)\right\|=0
$$


where the symbol $\|\ldots\|$ stands for determinant. Equation (19) yields an infinite set of roots $\left\{s_{n}\right\}$, each of which generates an eigenfunction. With a positive real part $\operatorname{Re} s_{n}$, the eigenfunction (12) has bounded elastic energy in any neighborhood of the notch tip, although this requirement doesn't exclude the existence of the stress singularity at $r=0$.

Finally, an inner Williams' expansion for the notch is given by

$$
\left(\begin{array}{c}
\mathbf{u}(r, \omega) \\
\boldsymbol{\phi}(r, \omega)
\end{array}\right)=\sum_{\operatorname{Re} s_{n} \geq 0} K^{(n) r^{s_{n}}} \hat{\mathbf{V}}^{\left(s_{n}\right)}(\omega)\left(\begin{array}{c}
\mathbf{h}_{n} \\
\mathbf{0}
\end{array}\right)
$$

with the coefficients $K^{(n)}$ being the eigenfunction amplitudes, which characterize the fine structure of the near-tip fields. Since the eigenvalue problem resulting from the traction free boundary conditions (17) is invariant with respect to the rigid body translations and rotations, the eigenvalues $s=0$ and $s=1$ are roots of equation (19), whatever the angular dependence the elastic constants $C_{i j k l}(\omega)$ have. Hence, two terms in Eq. (20) require special consideration. These two terms describe a rigid body translation and a rotation and they are ordered in the expansion (20) by $n=0$ and $n=1$ respectively. At this point, it is worth noting that the expansion coefficients $K^{(0)}$ and $K^{(1)}$ of both rigid body motion terms can be uniquely defined only if $S_{U} \neq 0$, where $S_{U}$ is a part of the body surface $S$ (see, Fig. 1 for details), at which the displacements are prescribed. Otherwise the two coefficients remain arbitrary and the corresponding terms in the expansion (20) can be omitted.

In the case of $S_{U} \neq 0$ the coefficients $K^{(0)}$ and $K^{(1)}$ become important, especially in the numerical analysis. In order to reveal their geometrical interpretation, let us consider the corresponding eigenfunctions explicitly. Rigid body translations are generated by the eigenvalue $s_{0}=0$. Since $\hat{\mathbf{V}}^{(0)}(\omega)$ reduces to the unit matrix, the eigenfunction associated with this eigenvalue takes the form

$$
\left(\begin{array}{l}
\mathbf{u}^{(0)}(r, \omega) \\
\boldsymbol{\phi}^{(0)}(r, \omega)
\end{array}\right)=K^{(0)}\left(\begin{array}{c}
\mathbf{h}_{0} \\
\mathbf{0}
\end{array}\right)
$$

Thereby the coefficient $K^{(0)}$ describes the notch tip (and the body as a whole) displacement magnitude in the direction of the vector $\mathbf{h}_{0}$, which length is assumed to be normalized to unity,

$$
\mathbf{u}(\mathbf{0})=K^{(0)} \mathbf{h}_{0} .
$$

In turn the rigid body rotation term is generated by the eigenvalue $s_{1}=1$ and the eigenvector $\mathbf{h}_{1}=\mathbf{n}(0)$. Using the properties of the ordered exponential $\hat{\mathbf{V}}^{(1)}(\omega)$ (consult with $[24,28]$ for further details), the corresponding eigenfunction can be found in explicit form for an arbitrary rotational inhomogeneity

$$
\left(\begin{array}{l}
\mathbf{u}^{(1)}(r, \omega) \\
\boldsymbol{\phi}^{(1)}(r, \omega)
\end{array}\right)=K^{(1)} r \hat{\mathbf{V}}^{(1)}(\omega)\left(\begin{array}{c}
\mathbf{n}(0) \\
\mathbf{0}
\end{array}\right)=\theta r\left(\begin{array}{c}
\mathbf{n}(\omega) \\
\mathbf{0}
\end{array}\right) .
$$

The coefficient $K^{(1)}=\theta$ represents a rigid body rotation by an angle $\theta$. 
In general, for some particular angular dependencies of the elastic constants $C_{i j k l}(\omega)$ or for some values of the notch angle the eigenvalue equation (19) can have multiple roots. This case needs special treatment since the expansion (20) over the power-law eigenfunctions is no longer complete and must be completed by the power-logarithmic solutions. The necessary modifications can be done by taking into consideration some general properties of solutions of elliptic problems in domains with piecewise smooth boundaries [26]. In fact, such degeneracies are of minor practical importance for fracture mechanics and are not discussed in this paper. The only exception is the root $s=1$ associated with rigid body rotation as well as the complementary root $s=-1$ generating a solution for a concentrated couple applied at the noth tip. This case is analyzed in detail in [29], where also analytical expressions for power-logarithmic solutions in elastically anisotropic angularly inhomogeneous media were presented (see also [24]).

\section{Complementary eigenfunctions}

The eigenfunction expansion (20) of the near-tip field contains only the terms of bounded elastic energy. However, the eigenvalue problem (18) admits solutions of unbounded energy as well. The latter correspond to self-equilibrated loadings applied at the notch tip. The eigenfunctions of bounded and unbounded elastic energy are not independent and there exists an intrinsic symmetry between them, which follows from the invariance of Eq. (19) with respect to the index inversion $s \rightarrow-s$. As has been proved by Belov and Kirchner [31], for any angular inhomogeneity $C_{i j k l}(\omega)$, whenever Eq. (19) is satisfied,

$$
\left\|\hat{\mathbf{V}}_{3}^{(-s)}(\Omega)\right\|=0
$$

is also valid. Hence, for any eigenfunction (12) generated by a positive real part root $s$ there exists a complementary eigenfunction generated by an eigenvalue $-s$ with negative real part and unbounded elastic energy. This symmetry between the positive and negative real part solutions of Eq. (19) is the cornerstone of the weight function theory.

Since eigenvalues $s$ and $-s$ appear always in pairs, the complementary eigenfunction

$$
\left(\begin{array}{l}
\mathbf{u}(r, \omega) \\
\boldsymbol{\phi}(r, \omega)
\end{array}\right)=r^{-s} \hat{\mathbf{V}}^{(-s)}(\omega)\left(\begin{array}{c}
\mathbf{h}^{*} \\
\mathbf{g}^{*}
\end{array}\right)
$$

where $\left(\mathbf{h}^{*}, \mathbf{g}^{*}\right)$ is a constant vector, is also a solution without body forces and dislocations that obeys the traction free boundary condition (17) at the notch faces. The vector $\left(\mathbf{h}^{*}, \mathbf{g}^{*}\right)$ excites this field just as (h, g) excited (12).

\section{Pseudo-orthogonality relations}

The second property of the eigenfunctions (12), which underlies the weight function theory, is their six-dimensional orthogonality. The paper by Chen [13] appears to be the first work where the orthogonality property of the eigenfunctions along with Betti's reciprocity theorem were applied to compute the coefficients in the Williams' eigenfunction expansion for an edge crack. The case an elastically isotropic medium considered in [13] is rather simple, since the existing analytical expressions for the eigenfunctions enable for the orthogonality property 
to be easily proved by direct calculation. Using the same method, Chen and Hasebe [25, 27] derived the orthogonality property for an interface crack in an isotropic bimaterial and also for an orthotropic material with pure imaginary roots of the Stroh matrix. The cumbersome direct calculations $[13,25,27]$ are possible only for very simple cases and reveal neither the nature of the orthogonality relations nor their connection with the symmetry of the elasticity equations. Belov and Kirchner [28] suggested a proof of the orthogonality property for both cracks and notches of finite opening angle in an elastically anisotropic media possessing arbitrary inhomogeneity of the elastic constants $C_{i j k l}(\omega)$. In contrast to [13, 25], the proof given in [28] shows that the orthogonality property of the eigenfunctions (12) directly follows from the symmetry (5) of the operator $\hat{\mathbf{N}}(\mathbf{r})$.

The idea of the proof [28] consists in the following. Integrating by parts an average of the weighted product of two ordered exponentials of arbitrary indices $s$ and $q$, one finds

$$
\begin{aligned}
q \int_{0}^{\Omega}\left[\hat{\mathbf{V}}^{(s)}(\omega)\right]^{\mathrm{t}} \hat{\mathbf{T}} \hat{\mathbf{N}}(\omega) \hat{\mathbf{V}}^{(q)}(\omega) \mathrm{d} \omega & =\int_{0}^{\Omega}\left[\hat{\mathbf{V}}^{(s)}(\omega)\right]^{\mathrm{t}} \hat{\mathbf{T}} \frac{\mathrm{d}}{\mathrm{d} \omega} \hat{\mathbf{V}}^{(q)}(\omega) \mathrm{d} \omega \\
& =\left[\hat{\mathbf{V}}^{(s)}(\Omega)\right]^{\mathrm{t}} \hat{\mathbf{T}} \hat{\mathbf{V}}^{(q)}(\Omega)-\hat{\mathbf{T}} \\
& -s \int_{0}^{\Omega}\left[\hat{\mathbf{V}}^{(s)}(\omega)\right]^{\mathrm{t}} \hat{\mathbf{N}}^{\mathrm{t}}(\omega) \hat{\mathbf{T}} \hat{\mathbf{V}}^{(q)}(\omega) \mathrm{d} \omega
\end{aligned}
$$

So far only the fact that the ordered exponential $\hat{\mathbf{V}}^{(q)}(\omega)$ satisfies equation (14) has been used. Taking into account that the 'bulk' operator $\hat{\mathbf{T}} \hat{\mathbf{N}}(\mathbf{r})$ is symmetric (according to Eq.(5)), we obtain an important property of the ordered exponentials

$$
(s+q) \int_{0}^{\Omega}\left[\hat{\mathbf{V}}^{(s)}(\omega)\right]^{\mathrm{t}} \hat{\mathbf{T}} \hat{\mathbf{N}}(\omega) \hat{\mathbf{V}}^{(q)}(\omega) \mathrm{d} \omega=\left[\hat{\mathbf{V}}^{(s)}(\Omega)\right]^{\mathrm{t}} \hat{\mathbf{T}} \hat{\mathbf{V}}^{(q)}(\Omega)-\hat{\mathbf{T}}
$$

This result is independent of the boundary conditions (17) specified at the notch faces. It takes place for any indices $s$ and $q$, which are not necessary roots of Eq. (19). Let us now consider two roots $s_{n}$ and $s_{p}$ satisfying the condition $s_{n}+s_{p} \neq 0$. Then, according to Eq. (27), the weighted average can be represented as

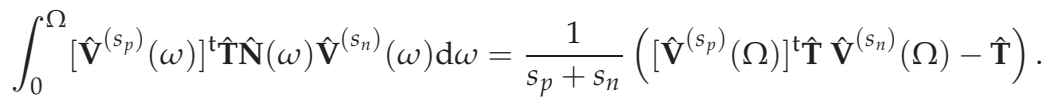

Now let $\left(\mathbf{h}_{p}, \mathbf{0}\right)$ and $\left(\mathbf{h}_{n}, \mathbf{0}\right)$ be corresponding eigenvectors. Multiplying equation (28) from the right and from the left by these eigenvectors, one obtains the orthogonality relation in the form

$$
\left(\mathbf{h}_{p}, \mathbf{0}\right)^{\mathrm{t}} \int_{0}^{\Omega}\left[\hat{\mathbf{V}}^{\left(s_{p}\right)}(\omega)\right]^{\mathrm{t}} \hat{\mathbf{T}} \hat{\mathbf{N}}(\omega) \hat{\mathbf{V}}^{\left(s_{n}\right)}(\omega) \mathrm{d} \omega\left(\begin{array}{c}
\mathbf{h}_{n} \\
\mathbf{0}
\end{array}\right)=0, \quad\left(s_{n}+s_{p} \neq 0\right) .
$$

Details of the proof are clear from Eq. (28) and the identity

$$
\left(\left[\hat{\mathbf{V}}^{\left(s_{p}\right)}(\Omega)\left(\begin{array}{c}
\mathbf{h}_{p} \\
\mathbf{0}
\end{array}\right)\right]^{\mathrm{t}} \hat{\mathbf{T}} \hat{\mathbf{V}}^{\left(s_{n}\right)}(\Omega)-\left(\mathbf{h}_{p}, \mathbf{0}\right) \hat{\mathbf{T}}\right)\left(\begin{array}{c}
\mathbf{h}_{n} \\
\mathbf{0}
\end{array}\right)=\left(\mathbf{h}_{p} \hat{\mathbf{V}}_{1}^{\left(s_{p}\right)}(\Omega), \mathbf{0}\right)\left(\begin{array}{c}
\mathbf{0} \\
\hat{\mathbf{V}}_{1}^{\left(s_{n}\right)}(\Omega) \mathbf{h}_{n}
\end{array}\right)=0 .
$$


Finally, the orthogonality relation can be rewritten explicitly in terms of the eigenfunctions (12) as

$$
\int_{0}^{\Omega}\left(\mathbf{u}_{p}, \boldsymbol{\phi}_{p}\right)^{\mathrm{t}} \hat{\mathbf{T}} \hat{\mathbf{N}}(\omega)\left(\begin{array}{c}
\mathbf{u}_{n} \\
\boldsymbol{\phi}_{n}
\end{array}\right) \mathrm{d} \omega=0, \quad\left(s_{n}+s_{p} \neq 0\right) .
$$

The six-dimensional orthogonality (or pseudo-orthogonality) is not an orthogonality in the generally accepted sense, because it takes place not only for different eigenvalues, but also when $s_{n}=s_{p}$. This means that all eigenfunctions are 'self-orthogonal'. The pseudo-orthogonality property fails only for the pairs $s_{p} \neq-s_{n}$, which have special status in the higher-order weight function theory.

\section{Fundamental field and weight function of higher-order}

Following [28], we consider a notched body $A$ shown in Fig. 1 and subject it to an external surface loading system which includes prescribed surface tractions $\mathbf{F}$ on the boundary $S_{\mathrm{T}}$ and imposed displacements $\mathbf{U}$ at the remainder $S_{\mathrm{U}}$ of the body surface $S=S_{\mathrm{T}}+S_{\mathrm{U}}$. We further suppose that $A$ is free from body forces and dislocations. The notch faces $S_{\mathrm{N}}$ are assumed to be traction free. This system of loadings leads to the boundary conditions

$$
\left\{\begin{array}{l}
T_{k}=\mathrm{F}_{k} \text { on } S_{\mathrm{T}}, \text { and } T_{k}=0 \text { on } S_{\mathrm{N}}, \\
u_{k}=\mathrm{U}_{k} \text { on } S_{\mathrm{U}},
\end{array}\right.
$$

where $T_{k}=\sigma_{i j} v_{j}$ and $v_{j}$ is an outer unit normal to the body surface. Inside $A$ the elastic field produced by the loading system (32) is represented by the eigenfunction expansion (20). This field is called regular, while it can result in a stress singularity at the notch tip. As already noted, the elastic energy associated with the regular field remains bounded in any neighborhood of the notch tip.

In order to derive weight functions for the coefficients $K^{(n)}$ in the series expansion (20), it is convenient to consider the cases $S_{U}=0$ and $S_{U} \neq 0$ separately.

(I) If $S_{\mathrm{U}}=0$, except for $n=0$ and 1 , all coefficients $K^{(n)}$ are defined uniquely. In order to find a coefficient $K^{(m)}$, one needs to apply Betti's reciprocity theorem to the regular field and to a specially chosen auxiliary field called the fundamental field of order $m$. It consists of a complementary solution (25) for the term of order $m$ and a regular part,

$$
\left(\begin{array}{c}
\mathbf{u}^{*(m)}(r, \omega) \\
\boldsymbol{\phi}^{*(m)}(r, \omega)
\end{array}\right)=r^{-s_{m}} \hat{\mathbf{V}}^{\left(-s_{m}\right)}(\omega)\left(\begin{array}{c}
\mathbf{h}_{m}^{*} \\
\mathbf{0}
\end{array}\right)+\sum_{\operatorname{Re} s_{p}>0} k_{p} r^{s_{p}} \hat{\mathbf{V}}^{\left(s_{p}\right)}(\omega)\left(\begin{array}{c}
\mathbf{h}_{p} \\
\mathbf{0}
\end{array}\right),
$$

where the sum is extended over all eigenfunctions of bounded elastic energy. The fundamental field of the $m$ th order corresponds to a certain source placed at $r=0$ and thereby provides zero body forces and dislocation density in the bulk. This justifies introduction of both the displacement (no dislocations) and the Airy stress function (no body forces) anywhere inside the body. The coefficients $k_{p}$ must be chosen so as to subject the solution (33) to the traction free boundary conditions

$$
T_{k}^{*(m)}=0 \quad \text { on } \quad S_{\mathrm{T}}+S_{\mathrm{N}} .
$$


Because $S_{U}=0$, the representation (33) of the $m$ th order fundamental field is possible for $m \neq 0$ and 1 . The terms corresponding to rigid body motions can be chosen arbitrary.

For a subdomain $A^{\prime} \subset A$, bounded as shown in Fig. 1 by a closed contour $L$ which consists of a circular arc $R_{0}$ of radius $r_{0}$ around the notch tip, the body surface $S=S_{\mathrm{T}}$, and the remaining part $S_{N^{\prime}}$ of the notch faces $S_{N}$, application of Betti's reciprocity theorem yields

$$
\Gamma^{(m)}=-\int_{S_{\mathrm{T}}+S_{\mathrm{N}^{\prime}}}\left(T_{k}^{*(m)} u_{k}-T_{k} u_{k}^{*(m)}\right) \mathrm{d} s=-\int_{S_{\mathrm{T}}} u_{k}^{*(m)} \mathrm{F}_{k} \mathrm{~d} s,
$$

where $u_{k}$ and $T_{k}$ are the displacement and traction due to the regular field, and

$$
\Gamma^{(m)}=\int_{R_{0}}\left(T_{k} u_{k}^{*(m)}-T_{k}^{*(m)} u_{k}\right) \mathrm{d} s .
$$

Integrating Eq.(36) by part, one obtains

$$
\Gamma^{(m)}=\boldsymbol{\phi}^{*(m)}(r, 0) \mathbf{u}^{*(m)}(r, 0)-\boldsymbol{\phi}^{*(m)}(r, \Omega) \mathbf{u}^{*(m)}(r, \Omega)+\int_{0}^{\Omega}\left(\boldsymbol{\phi}^{*(m)}, \mathbf{u}^{*(m)}\right) \frac{d}{d \omega}\left(\begin{array}{l}
\mathbf{u} \\
\boldsymbol{\phi}
\end{array}\right) \mathrm{d} \omega,
$$

with the two first terms vanishing owing to the traction free boundary conditions (17) imposed on all eigenfunctions at the notch faces. Hence, substituting the explicit expressions for the regular and fundamental field into (37), one finds

$$
\begin{aligned}
\Gamma^{(m)} & =\sum_{n} K^{(n)} s_{n} r_{0}^{s_{n}-s_{m}}\left(\mathbf{h}_{m}^{*}, \mathbf{0}\right)^{\mathrm{t}} \int_{0}^{\Omega}\left[\hat{\mathbf{V}}^{\left(-s_{m}\right)}(\omega)\right]^{\mathrm{t}} \hat{\mathbf{T}} \hat{\mathbf{N}}(\omega) \hat{\mathbf{V}}^{\left(s_{n}\right)}(\omega) \mathrm{d} \omega\left(\begin{array}{c}
\mathbf{h}_{n} \\
\mathbf{0}
\end{array}\right) \\
& +\sum_{n, p} K^{(n)} k_{p} s_{n} r_{0}^{s_{n}+s_{p}}\left(\mathbf{h}_{p}, \mathbf{0}\right)^{\mathrm{t}} \int_{0}^{\Omega}\left[\hat{\mathbf{V}}^{\left(s_{p}\right)}(\omega)\right]^{\mathrm{t}} \hat{\mathbf{T}} \hat{\mathbf{N}}(\omega) \hat{\mathbf{V}}^{\left(s_{n}\right)}(\omega) \mathrm{d} \omega\left(\begin{array}{c}
\mathbf{h}_{n} \\
\mathbf{0}
\end{array}\right) .
\end{aligned}
$$

As $r_{0}$ shrinks to zero, the second sum in Eq. (38) vanishes due to the fact that the real parts of all eigenvalues $s_{n}$ and $s_{p}$ are positive. However, there is also another reason for this term in Eq. (38) to vanish. In fact, it must vanish due to the pseudo-orthogonality property (29). As concerns the first sum in Eq. (38), it contains the terms formally divergent as the radius $r_{0} \rightarrow 0$. However, owing to the pseudo-orthogonality property (29), these terms drop out of Eq. (38) and finally only one term for which $s_{n}=s_{m}$ remains non-vanishing. This term is independent of $r_{0}$ and remains constant as it shrinks. Note also that the second sum in Eq. (38) is not sensitive to the rigid body motion terms in the fundamental field.

According to Eq. (38), the reciprocity theorem relates the expansion coefficient of order $m$ directly with external loading as

$$
K^{(m)} Y^{(m)}=-\int_{S_{\mathrm{T}}} u_{k}^{*(m)} \mathrm{F}_{k} \mathrm{~d} s,
$$

with a normalizing geometry factor

$$
Y^{(m)}=-s_{m}\left(\mathbf{h}_{m}^{*}, \mathbf{0}\right)^{\mathrm{t}} \int_{0}^{\Omega}\left[\hat{\mathbf{V}}^{\left(-s_{m}\right)}(\omega)\right]^{\mathrm{t}} \hat{\mathbf{T}} \hat{\mathbf{N}}(\omega) \hat{\mathbf{V}}^{\left(s_{m}\right)}(\omega) \mathrm{d} \omega\left(\begin{array}{c}
\mathbf{h}_{m} \\
\mathbf{0}
\end{array}\right)
$$


Correspondingly, an expansion coefficient $K^{(m)}$ is available via the $m$ th order weight function,

$$
h_{k}^{(m)}\left(x_{1}, x_{2}\right)=u_{k}^{*(m)}\left(x_{1}, x_{2}\right) / Y^{(m)},
$$

as a functional

$$
K^{(m)}=\int_{S_{\mathrm{T}}} h_{k}^{*(m)}\left(x_{1}, x_{2}\right) \mathrm{F}_{k} \mathrm{~d} s
$$

of the surface loading. Thus the $m$ th order weight function differs from the corresponding fundamental field (33) only in a constant geometry factor (40).

(II) If $S_{U} \neq 0$, all expansion coefficients in the series (20) are defined unambiguously, including $K^{(0)}$ and $K^{(1)}$. For $m \neq 0$ the fundamental field of the $m$ th order is still given by the solution (33) provided that its bounded energy part is completed by the rigid body motion terms. The coefficients $k_{p}$ in (33) are now chosen to subject it to the boundary conditions

$$
\left\{\begin{array}{lll}
T_{k}^{*(m)}=0 & \text { on } & S_{\mathrm{T}}+S_{\mathrm{N}} \\
u_{k}^{*(m)}=0 & \text { on } & S_{\mathrm{U}} .
\end{array}\right.
$$

Modifying the reciprocal relation (35) to include $S_{U}$, one obtains

$$
\Gamma^{(m)}=-\int_{S_{\mathrm{T}}} u_{k}^{*(m)} \mathrm{F}_{k} \mathrm{~d} s+\int_{S_{\mathrm{T}}} T_{k}^{*(m)} \mathrm{U}_{k} \mathrm{~d} s .
$$

The remaining calculations are similar to those performed in the case of vanishing $S_{U}$. Weight functions of the $m$ th order are introduced according to

$$
\begin{aligned}
& h_{k}^{(m)}\left(x_{1}, x_{2}\right)=u_{k}^{*(m)}\left(x_{1}, x_{2}\right) / Y^{(m)}, \\
& H_{k}^{(m)}\left(x_{1}, x_{2}\right)=T_{k}^{*(m)}\left(x_{1}, x_{2}\right) / Y^{(m)} .
\end{aligned}
$$

The coefficients $K^{(m)}$ in the eigenfunction expansion are now expressed via the $m$ th order weight functions as

$$
K^{(m)}=\int_{S_{\mathrm{T}}} h_{k}^{*(m)}\left(x_{1}, x_{2}\right) \mathrm{F}_{k} \mathrm{~d} s-\int_{S_{\mathrm{U}}} H_{k}^{*(m)}\left(x_{1}, x_{2}\right) \mathrm{U}_{k} \mathrm{~d} s .
$$

As it was noted above, in the case of non-vanishing $S_{U}$ the coefficient $K^{(0)}$ needs a special treatment. The complementary field for the rigid body translation (21) has a logarithmic rather than power-law functional form. Indeed, the auxiliary source generating this complementary solution is a concentrated force applied at the notch tip. Unlike other eigenfunctions it is not self-equilibrated. The complementary logarithmic solution can be constructed by means of the analytical expression for the elastic field of a force at the tip of a notch in an angularly inhomogeneous plane [16, 18]. Details of further development and the corresponding 0th order fundamental field for the calculation of the rigid body translation term are available from [28]. 


\section{Multimaterials}

A continuously inhomogeneous elastic material is actually only a useful tool, which considerably simplifies the establishing of important properties of elastic fields involved in weight function theory. Nowadays functionally graded materials with continuous angular inhomogeneity of elastic properties are still exotic and in engineering structures we deal mostly with piecewise homogeneous media (junctions of a finite number of dissimilar materials) called multimaterials. In the case of multimaterials further analytical advancement in the weight function theory becomes possible. The ordered exponentials are known to appear in Eq. (16) instead of the conventional exponentials since the angular inhomogeneity causes non-commutability of the matrices $\hat{\mathbf{N}}(\omega)$ for different values of the argument $\omega$. However, when the medium is piecewise homogeneous, the matrices $\hat{\mathbf{N}}(\omega)$ commute within each homogeneous wedge-like region [15] and the integration in the ordered exponentials can be performed analytically. For example, in the case of a multimaterial composed from three wedges (triple junction)

$$
C_{i j k l}(\omega)=\left\{\begin{array}{lll}
C_{i j k l}^{(1)} & & 0<\omega<\alpha, \\
C_{i j k l}^{(2)} & \text { for } \quad & \alpha<\omega<\beta, \\
C_{i j k l}^{(3)} & & \beta<\omega<\Omega,
\end{array}\right.
$$

the ordered exponential admits factorization and reduces to (for details, see [17, 23, 24])

$$
\operatorname{Ordexp}\left(s \int_{0}^{\omega} \hat{\mathbf{N}}(\theta) \mathrm{d} \theta\right)=\left\{\begin{array}{lr}
\hat{\mathbf{V}}_{1}^{s}(\omega) & 0<\omega<\alpha, \\
\hat{\mathbf{V}}_{2}^{s}(\omega) \hat{\mathbf{V}}_{2}^{-s}(\alpha) \hat{\mathbf{V}}_{1}^{s}(\alpha) & \text { for } \quad \alpha<\omega<\beta, \\
\hat{\mathbf{V}}_{3}^{s}(\omega) \hat{\mathbf{V}}_{3}^{-s}(\beta) \hat{\mathbf{V}}_{2}^{s}(\beta) \hat{\mathbf{V}}_{2}^{-s}(\alpha) \hat{\mathbf{V}}_{1}^{s}(\alpha) & \beta<\omega<\Omega,
\end{array}\right.
$$

where $\hat{\mathbf{V}}_{i}^{s}(\omega)$ and $\hat{\mathbf{V}}_{i}^{-s}(\omega)$ denote for each homogeneous region powers of

$$
\hat{\mathbf{V}}_{i}(\omega)=\operatorname{Ordexp}\left(\int_{0}^{\omega} \hat{\mathbf{N}}(\theta) \mathrm{d} \theta\right)=\hat{\mathbf{I}} \cos \omega+\hat{\mathbf{N}}_{i}(0) \sin \omega
$$

and

$$
\hat{\mathbf{V}}_{i}^{-1}(\omega)=\hat{\mathbf{I}} \cos \omega-\hat{\mathbf{N}}_{i}(\omega) \sin \omega,
$$

The matrix $\hat{\mathbf{N}}_{i}(\omega)$ for each homogeneous wedge-like region of a multimaterial is constructed by replacing $C_{i j k l}(\omega)$ in the definition (4) by $C_{i j k l}^{(i)}$. If $s$ is not an integer, the powers of the matrices (50) and (51) should be defined in terms of their spectral decompositions over the eigenvectors of the matrices $\hat{\mathbf{N}}_{i}(\omega)$ (for details, see [22]).

\section{Conclusions}

Here, it was shown that the established in [28] pseudo-orthogonality property of the power eigenfunctions follows directly from the symmetry of the operator $\hat{\mathbf{N}}(\mathbf{r})$, which is commonly referred to as Stroh matrix [3, 22] of anisotropic elasticity theory. In the last decade the proof of the pseudo-orthogonality property was republished in a large number of papers [32-38], where however only trivial particular cases of anisotropy and inhomogeneity were 
analyzed. The general proof by Belov and Kirchner [28] is not cited in these papers, which are to be considered as plagiarism, although some of them contain further development, in particular, by taking into account piezoelectricity. Here, it is worth to mention that the proof of the pseudo-orthogonality property remains valid for the general case of piezoelectric piezomagnetic magnetoelectric anisotropic media, provided that the dimension of both the matrix $\hat{\mathbf{N}}(\mathbf{r})$ and the field variables is increased to include these effects (for details, see [30]). In conclusion, it may be also said that the pseudo-orthogonality property allows for a set of path-independent integrals similar to $H$-integral $[7,8,10-12]$ to be introduced for multimaterials with notches or cracks. This is achieved by applying Betti's reciprocity theorem to the complementary field (25) rather than to the fundamental field (33). The contour $L$ must be properly shifted from the surface $S$ to interior domain of $A$.

\section{Author details}

Alexander Yu. Belov

Institute of Crystallography RAS, Moscow, Russian Federation

\section{References}

[1] Williams, M.L. (1952). Stress singularities resulting from various boundary conditions in angular corners of plates in extension, ASME Journal of Applied Mechanics, 19:526-528.

[2] Williams, M.L. (1957). On the stress distribution at the base of a stationary crack, ASME Journal of Applied Mechanics, 24:109-114.

[3] Stroh, A.N. (1962). Steady State Problems in Anisotropic Elasticity, Journal of Mathematical Physics, 41:77-103.

[4] Bueckner, H.F. (1970). A Novel Principle for the Computation of Stress Intensity Factors. Zeitschrift für Angewandte Mathematik und Mechanik, 50:529-546.

[5] Malén, K. \& Lothe, J. (1970). Explicit Expressions for Dislocation Derivatives. Physica Status Solidi, 39:287-296.

[6] Bueckner, H.F. (1971). Weight Functions for the Notched Bar. Zeitschrift für Angewandte Mathematik und Mechanik, 51:97-109.

[7] Stern, M.; Becker, E.H. \& Dunham, R.S. (1976). A Contour Integral Computation of Mixed-Mode Stress Intensity Factors. International Journal of Fracture, 12:359-368.

[8] Stern, M. \& Soni, M.L. (1976). On the Computation of Stress Intensities at Fixed-Free Corners. International Journal of Solids and Structures, 12:331-337.

[9] Chadwick, P. \& Smith, G.D. (1977). Foundations of the Theory of Surface Waves in Anisotropic Elastic Materials. Advances in Applied Mechanics, 17:303-376.

[10] Hong, C.-C. \& Stern, M. (1978). The Computation of Stress Intensity Factors in Dissimilar Materials. Journal of Elasticity, 8:21-34.

[11] Stern, M. (1979). The Numerical Calculation of Thermally Induced Stress Intensity Factors. Journal of Elasticity, 9:91-95.

[12] Sinclair, G.B.; Okajima, M. \& Griffin, J.H. (1984). Path Independent Integrals for Computing Stress Intensity Factors at Sharp Notches in Elastic Plates. International Journal for Numerical Methods in Engineering, 20:999-1008. 
[13] Chen, Y.Z. (1986). New Path Independent Integrals in Linear Elastic Fracture Mechanics. Engineering Fracture Mechanics, 22:673-686.

[14] Kirchner, H.O.K. (1986). Description of loadings and screenings of cracks with the aid of universal weight functions. International Journal of Fracture, 31:173-181.

[15] Kirchner, H.O.K. \& Lothe, J. (1986). On the redundancy of the $\bar{N}$ matrix of anisotropic elasticity. Philosophical Magazine Part A, 53:L7-L10.

[16] Kirchner, H.O.K. (1987). Line defects along the axis of rotationally inhomogeneous media. Philosophical Magazine Part A, 55:537-542.

[17] Kirchner, H.O.K. (1989). Elastically anisotropic angularly inhomogeneous media I. A new formalism. Philosophical Magazine Part B, 60:423-432.

[18] Ting, T.C.T. (1989). Line forces and dislocations in angularly inhomogeneous anisotropic elastic wedges and spaces. Quaterly Applied Mathematics, 47:123-128.

[19] Bueckner, H.F. (1989). Observations on weight functions. Engineering Analysis with Boundary Elements, 6:3-18.

[20] Sham, T.L. (1989). The theory of higher order weight functions for linear elastic plane problems. International Journal of Solids and Structures, 25:357-380.

[21] Sham, T.L. (1991). The determination of the elastic T-term using higher order weight functions. International Journal of Fracture, 48:81-102.

[22] Indenbom, V.L. \& Lothe, J. (Eds.) (1992). Elastic Strain Fields and Dislocation Mobility, Amsterdam: North-Holland.

[23] Belov, A.Yu. (1992). A wedge disclination along the vertex of the wedge-like inhomogeneity in an elastically anisotropic solid. Philosophical Magazine Part A, 65:1429-1444.

[24] Belov, A.Yu. (1993). Scaling regimes and anomalies of wedge disclination stresses in anisotropic rotationally inhomogeneous media. Philosophical Magazine Part A, 68:1215-1231.

[25] Chen, Y.Z. \& Hasebe, N. (1994). Eigenfunction Expansion and Higher Order Weight Functions of Interface Cracks. ASME Journal of Applied Mechanics 61:843-849.

[26] Nazarov, S.A. \& Plamenevsky, B.A. (1994). Elliptic Problems in Domains with Piecewise Smooth Boundaries, volume 13 of de Gruyter Expositions in Mathematics. Berlin, New York: Walter de Gruyter and Co.

[27] Chen, Y.H. \& Hasebe, N. (1995). Investigation of EEF Properties for a Crack in a Plane Orthotropic Elastic Solid with Purely Imaginary Characteristic Roots. Engineering Fracture Mechanics, 50:249-259.

[28] Belov, A.Yu. \& Kirchner, H.O.K. (1995). Higher order weight functions in fracture mechanics of inhomogeneous anisotropic solids. Philosophical Magazine Part A, 72:1471-1483.

[29] Belov, A.Yu. \& Kirchner, H.O.K. (1995). Critical angles in bending of rotationally inhomogeneous elastic wedges. ASME Journal of Applied Mechanics, 62: 429-440.

[30] Alshits, V.I.; Kirchner, H.O.K. \& Ting, T.C.T. (1995). Angularly inhomogeneous piezoelectric piezomagnetic magnetoelectric anisotropic media. Philosophical Magazine Letters, 71:285-288.

[31] Belov, A.Yu. \& Kirchner, H.O.K. (1996). Universal weight functions for elastically anisotropic, angularly inhomogeneous media with notches or cracks. Philosophical Magazine Part A, 73:1621-1646. 
[32] Qian, J. \& Hasebe, N. (1997). Property of Eigenvalues and Eigenfunctions for an Interface V-Notch in Antiplane Elasticity. Engineering Fracture Mechanics, 56:729-734.

[33] Chen, Y.H. \& Ma, L.F. (2000). Bueckner's Work Conjugate Integrals and Weight Functions For a Crack in Anisotropic Solids. Acta Mechanica Sinica (English Series), $16: 240-253$

[34] Ma, L.F. \& Chen, Y.H. (2001). Weight Functions for Interface cracks in Dissimilar Anisotropic Piezoelectric Materials. International Journal of Fracture 110:263-279.

[35] Chen, Y.H. \& Ma, L.F. (2004). Weight Functions for Interface Cracks in Dissimilar Anisotropic Materials. Acta Mechanica Sinica (English Series), 16:82-88.

[36] Ou, Z.C. \& Chen, Y.H. (2004). A New Method for Establishing Pseudo Orthogonal Properties of Eigenfunction Expansion Form in Fracture Mechanics. Acta Mechanica Solida Sinica, 17:283-289.

[37] Ou, Z.C. \& Chen, Y.H. (2006). A New approach to the Pseudo-Orthogonal Properties of Eigenfunction Expansion Form of the Crack-Tip Complex Potential Function in Anisotropic and Piezoelectric Fracture Mechanics. European Journal of Mechanics A/Solids, 25:189-197.

[38] Klusák, J.; Profant, T. \& Kotoul, M. (2009). Various Methods of Numerical Estimation of Generalized Stress Intensity Factors of Bi-Material Notches. Applied and Computational Mechanics, 3:297-304. 